\title{
Apoio matricial em saúde mental infantojuvenil na Atenção Primária à Saúde: pesquisa intervenção socioclínica institucional*
}

\author{
Matrix support in children's mental health in Primary Health Care: \\ institutional socio-clinical intervention research \\ Matriz de apoyo en salud mental infantil en Atención Primaria de Salud: \\ investigación de intervención socio-clínica institucional
}

Como citar este artigo:

Oliveira PS, Santana FR, Gatto Júnior JR, Santos KS, Araujo PN, Fortuna CM. Matrix support in children's mental health in Primary Health Care: institutional socio-clinical intervention research. Rev Esc Enferm USP. 2021;55:e03731. https://doi.org/10.1590/S1980-220X2020016803731

\section{Poliana Silva de Oliveira ${ }^{1}$ \\ Fabiana Ribeiro Santana² \\ José Renato Gatto Júnior ${ }^{3}$ \\ Karen da Silva Santos ${ }^{4}$ \\ Priscila Norié de Araujo ${ }^{4}$ \\ Cinira Magali Fortuna ${ }^{5}$}

\footnotetext{
* Extraído da dissertação: "Experimentações no apoio matricial em saúde mental para crianças e adolescentes na atenç̃o básica: a busca pela aprendizagem coletiva”, Programa Pós-Graduação Mestrado Profissional de Tecnologia e Inovação em Enfermagem, Escola de Enfermagem de Ribeirão Preto, Universidade de São Paulo, 2018.

${ }^{1}$ Universidade de São Paulo, Escola de Enfermagem de Ribeirão Preto,

Programa Pós-Graduação Mestrado Profissional de Tecnologia e Inovação em

Enfermagem, Ribeirão Preto, SP, Brasil.

${ }^{2}$ Universidade Federal de Goiás, Instituto de Patologia Tropical e Saúde Pública, Departamento de Saúde Coletiva, Goiânia, GO, Brasil.

${ }^{3}$ Faculdade de Educação São Luís, Ribeirão Preto, SP, Brasil.

${ }^{4}$ Universidade de São Paulo, Escola de Enfermagem de Ribeirão Preto, Programa de Pós-Graduação Enfermagem em Saúde Pública, Ribeirão Preto, SP, Brasil.

${ }^{5}$ Universidade de São Paulo, Escola de Enfermagem de Ribeirão Preto,

Departamento Materno-Infantil e Saúde

Pública, Ribeirão Preto, SP, Brasil.
}

\begin{abstract}
Objective: To analyze matrix support for Family Health Strategy teams in relation to Mental Health in Children and Adolescents. Method: This is a research-intervention with a qualitative approach, based on the Institutional Analysis framework, Socio-clinic, carried out with eighteen health workers from two Family Health Strategy and Psychosocial Care Center teams of a small municipality in the countryside of the state of São Paulo, through eleven reflection meetings. Results: The following themes emerged: The dynamics of relations in the FHS territory; Matrix Support as a technological device: unveiling established practices. Subsequently, the results were discussed based on the principles of Institutional Socio-clinic. Conclusion: Matrix support in children's mental health, based on Institutional Socio-clinic, favored the deterritorialization of professionals, revealing how mental health care is provided for children and adolescents, and the crossings that occur in the production of this care as well as possible paths to be followed to improve health actions.
\end{abstract}

\section{DESCRIPTORS}

Nursing; Mental Health; Family Health Strategy; Patient Care Team; Professional Practice.

\section{Autor correspondente:}

Cinira Magali Fortuna

Rua Carlos de Lima, 290, Royal

Park Bonfim Paulista

CEP 14028-560 - Ribeirão Preto, SP, Brasil

fortuna@eerp.usp.br 


\section{INTRODUÇÃO}

As Reformas Sanitária e Psiquiátrica, ocorridas durante as décadas de 70-80, alteraram a forma de atenção à saúde mental no contexto brasileiro. A assistência, antes centralizada no Hospital Psiquiátrico, passa a ser direcionada a serviços de base territorial, com valorização da reinserção social, práticas de cuidado centradas nas pessoas em seu modo de viver e não somente nos sintomas relacionados a algum problema psiquiátrico ${ }^{(1)}$. Particularmente em relação ao público infantojuvenil, a criação do Sistema Único de Saúde (SUS), em 1988, e a promulgação do Estatuto da Criança e do Adolescente, em 1990, permitiram que crianças e adolescentes fossem considerados como sujeitos de direito, valorizando o modo singular que estes apresentam de ser e estar no mundo, resguardando o direito universal de acesso à saúde e à educação ${ }^{(1)}$.

Em 2004, o Fórum Nacional de Saúde Mental Infantojuvenil promoveu a discussão entre vários setores de assistência e proteção (saúde, educação, justiça, assistência social) e a sociedade civil sobre a atenção à Saúde Mental a Crianças e Adolescentes (SMCA), apontando como estratégias fundamentais e desejáveis a articulação entre os serviços específicos de saúde mental, como os Centros de Atenção Psicossociais (CAPS) e a Atenção Primária à Saúde (APS), representada principalmente pela Estratégia Saúde da Família $(\mathrm{ESF})^{(2)}$.

As discussões do referido fórum eram condizentes com a lógica de atenção defendida pela Organização Mundial da Saúde (OMS) e dos movimentos das Reformas Psiquiátrica e Sanitária, que priorizam o cuidado em saúde mental como qualidade de vida do público infantojuvenil ${ }^{(2)}$.

No desenvolvimento do cuidado à SMCA na ESF, é relevante o estabelecimento de relacionamento terapêutico com crianças, adolescentes e suas famílias, em especial aqueles em situação de vulnerabilidade social ou em contexto de violência ${ }^{(3)}$. No entanto, ainda é um desafio o reconhecimento que o cuidado à SMCA deve estar presente em todas ações realizadas ao público infantojuvenil, não somente nos casos em que a criança/adolescente já apresenta algum problema psiquiátrico/emocional ${ }^{(4)}$.

A articulação entre as equipes da ESF e CAPS é necessária para o desenvolvimento de um cuidado integral à população infantojuvenil. Contudo, tem sido um desafio o estabelecimento da articulação efetiva entre estas equipes ${ }^{(5)}$.

São relevantes as estratégias que permitem aos profissionais olharem para sua prática, repensarem seu trabalho, produzindo novos sentidos e significados. Dessa forma, destacamos, neste estudo, o apoio matricial (AM) $)^{(6)}$ como uma estratégia utilizada para a aproximação entre a APS e a saúde mental ${ }^{(5)}$.

O AM é considerado uma tecnologia em saúde, classificada como híbrida, ao intervir no campo das tecnologias leves e leve-duras ${ }^{(7)}$. Ele oportuniza a reestruturação dos padrões das relações de trabalho em saúde, o encontro dos trabalhadores com outros trabalhadores e com os usuários, contribuindo como os profissionais/equipes estabelecem a gestão do cuidado que realizam, por exemplo, com vistas a práticas interdisciplinares e interprofissionais ${ }^{(6-7)}$.

Em geral, o apoio é realizado por algum profissional que auxilia as equipes no desenvolvimento de suas ações. Pode ocorrer discussões de casos, formulação coletiva de projeto terapêutico singular (PTS), visitas domiciliares e atendimentos conjuntos. Nessa perspectiva, o AM utiliza conceitos de campo e núcleo, pois profissionais de diferentes núcleos de saber trocam conhecimentos entre si para a construção de um campo adequado de práticas. Cada profissão, por meio de uma relação democrática, com seus saberes e práticas, procura apoiar e dialogar para um objetivo em comum: a resolubilidade e integralidade da atenção ${ }^{(6)}$. O AM pode ser desenvolvido em várias ações de cuidado em saúde, principalmente relacionado à APS.

Entretanto, ainda persistem desafios práticos para uma consolidação plena do AM como ferramenta de cuidado ampliado, sobretudo no campo de atuação da $\operatorname{SMCA}^{(5)}$. Esses desafios envolvem uma compreensão e exercício do $\mathrm{AM}$ próximo à prática do encaminhamento/triagem ou como atendimento pontual de casos que abarcam questões específicas em saúde mental ${ }^{(5)}$.

O enfermeiro é um importante membro da equipe do CAPS e da ESF, pois desempenha funções que incluem desde a coordenação e a articulação das equipes e entre serviços até o lugar de agente terapêutico no cuidado direto aos usuários. Aposta-se, assim, no potencial desse profissional no desenvolvimento do AM e da integração e articulação dessas equipes e campos específicos (CAPS e ESF).

Diante desse contexto, no intento de inspirar novas práticas, este estudo propôs uma pesquisa-intervenção com o objetivo de analisar o AM para equipes da ESF em relação à SMCA. Toma-se como questão norteadora: como o AM orientado pelos princípios da Socioclínica Institucional pode contribuir para realização de cuidados compartilhados entre o CAPS e a ESF visando à SMCA?

\section{MÉTODO}

\section{TIPO DO ESTUDO}

Trata-se de uma pesquisa-intervenção de abordagem qualitativa, sustentada pelo referencial teórico-metodológico da Análise Institucional, linha Socioclínica Institucional ${ }^{(8)}$.

A Análise Institucional é uma das correntes do Movimento Institucionalista, cujo objetivo é provocar processos de autogestão e autoanálise em coletivos $^{(9)}$. A Socioclínica Institucional, elaborada por Gilles Monceau, é um desdobramento aprimorado da Socioanálise, vertente interventiva da Análise Institucional, fundamentada por René Lourau e Georges Lapassade. A Socioclínica tem como objetivo a produção de conhecimentos e a análise coletiva das implicações profissionais, levando em consideração as subjetividades dos agentes sociais, os seus encontros e desencontros ${ }^{(8,10)}$. A mesma tem se apresentado como um importante referencial para pesquisas em enfermagem e saúde coletiva ${ }^{(10)}$. 
Gilles Monceau ${ }^{(8)}$ elaborou oito princípios norteadores do método de intervenção Socioclínica, sendo eles: "Análise da encomenda e das demandas"; "Participação dos sujeitos no dispositivo"; "Trabalho dos analisadores"; "Análise das transformações que se produzem à medida que o trabalho avança”; "Aplicação de modalidades de restituição"; "Análise das implicações primárias e das implicações secundárias"; "Intenção da produção de conhecimentos"; "Atenção aos contextos e as interferências institucionais"(8).

\section{População}

O estudo ocorreu em um município de pequeno porte, situado no interior do estado de São Paulo, onde não havia iniciativas efetivas de AM em saúde mental. A articulação entre o CAPS e a ESF era frágil, sendo realizada comumente por meio de encaminhamentos de papel.

O estudo foi produzido com profissionais de saúde de duas equipes da ESF e do CAPS, sendo separados em dois grupos (A e B). Cada grupo foi formado por profissionais de uma equipe da ESF e um trabalhador do CAPS (escolhido pela coordenadora do CAPS). No grupo A, participaram uma enfermeira, uma técnica de enfermagem, quatro agentes comunitárias de saúde (ACS), uma dentista da equipe da ESF e uma psicóloga do CAPS. No grupo B, participaram uma psicóloga representante do CAPS, uma enfermeira, duas técnicas de enfermagem, um dentista e cinco ACS da ESF. Consideraram-se como possíveis participantes os trabalhadores que não estivessem afastados de suas funções por motivos de licença ou problemas de saúde e tivessem disponibilidade para participar dos encontros realizados.

Em uma pesquisa-intervenção, efetuou-se uma coprodução dos dados entre o pesquisador e participantes da pesquisa, sustentada pela premissa de transformar para conhecer ${ }^{(11)}$. Desse modo, o pesquisador, além de promover a intervenção, é também integrante efetivo dela ${ }^{(12)}$. A primeira autora deste estudo participou como coordenadora dos encontros de reflexão. Ela é enfermeira de um CAPS Infantil, mestre em ciências pelo mestrado profissional em enfermagem e membro de um grupo de estudos sobre Análise Institucional.

\section{Produção E ANÁLISE DOS DADOS}

Realizaram-se encontros de reflexão, entendidos como espaços de fala e escuta compartilhados entre pesquisadores e participantes da pesquisa e que se pautam em uma dupla ambição: produzir conhecimentos científicos e o desenvolvimento profissional dos participantes ${ }^{(13)}$. O propósito dos encontros foi promover a discussão e análise de aspectos e práticas concernentes ao campo da SMCA na APS.

Nos encontros de reflexão, foi discutida a forma como os participantes gostariam de realizar as discussões, por meio de temas ou casos. Os profissionais optaram pelo emprego de temas disparadores, ora escolhidos pela pesquisadora e ora por eles. As discussões temáticas foram abrangentes e acabaram incluindo a discussão de casos de crianças/adolescentes acompanhados pelas equipes. Os principais temas trabalhados foram: o trabalho desenvolvido na ESF e CAPS com a população infantojuvenil; o cuidado a familiares de pessoas com intenso sofrimento psíquico; as possibilidades de atuação no programa saúde na escola; a prevenção ao suicídio na adolescência. Em todos os encontros, buscou-se incentivar a inclusão do debate sobre casos e situações concretas do cotidiano dos profissionais e equipes.

Além disso, ocorreram encontros de restituição ${ }^{(12)}\left(3^{\circ}\right.$ e $6^{\circ}$ encontros do grupo $\mathrm{A} ; 2^{\circ}$ e $5^{\circ}$ encontros do grupo B) por meio da leitura de sínteses feitas pela pesquisadora com apontamentos e análises das discussões realizadas nos encontros anteriores.

Conjuntamente, foram utilizadas as anotações do diário de pesquisa da primeira autora do estudo como ferramenta de apoio à intervenção.

A produção de dados ocorreu no período de agosto a setembro de 2017 por meio da realização de 11 encontros de reflexão entre os participantes da pesquisa. Ocorreram seis encontros com os membros do grupo A, em uma sala de reunião da primeira unidade da ESF, e cinco encontros com os membros do grupo B, em uma sala de reunião da segunda unidade. Os encontros tiveram duração de 60 a 120 minutos, sendo gravados em mídia digital e transcritos na íntegra. Os dados foram ordenados para a análise de acordo com os processos de transcrição, transposição e reconstituição ${ }^{(14)}$, e foram analisados tendo por referência a Socioclínica Institucional ${ }^{(8)}$.

\section{Aspectos ÉTICOS}

A pesquisa está em conformidade com a Resolução $\mathrm{n}^{\circ}$ 466/12 do Conselho Nacional de Saúde e foi aprovada pelo Comitê de Ética em Pesquisa sob CAAE no 640.02117.2.0000.5393. Realizou-se o contato com os participantes para apresentação dos objetivos, percurso metodológico e aspectos éticos para a produção do estudo, momento no qual assinaram o Termo de Consentimento Livre e Esclarecido (TCLE). Informou-se que a primeira autora realizaria a coordenação dos encontros da pesquisa, apesar de não possuir relação de chefia ou hierarquia com os trabalhadores de saúde do município estudado. Os encontros de reflexão ocorreram em local e horário sugerido por cada grupo de profissionais.

\section{RESULTADOS}

A apresentação dos resultados foi realizada a partir de dois temas: A dinâmica das relações no território da ESF; $O$ apoio matricial como dispositivo tecnológico: desvelando práticas instituídas.

$\mathrm{O}$ primeiro intencionou retratar as relações estabelecidas entre os trabalhadores, a população, os setores e equipamentos de saúde da área de abrangência da ESF e do município. O segundo diz respeito ao processo de desterritorialização dos participantes provocado pelo dispositivo AM, sustentado pelo referencial da Socioclínica.

\section{A DINÂMICA DAS RELAÇÕES NO TERRITÓRIO DA ESF}

Nos encontros, foram realizadas rodas de conversa, com uma participação ativa e análises coletivas das equipes, possibilitando o relato de suas experiências e a aproximação com a prática do cuidado a SMCA. 
(...) às vezes, eu vejo, assim, um adolescente que você percebe que não tá normal, mas a mãe fala assim "Ah, ele é assim mesmo, estranho, não conversa com ninguém”. Eu tenho medo de ela achar que eu tô invadindo o espaço dele sabe, depois querer me afastar, acabar não recebendo do jeito que recebe (...) teve um caso na minha área de uma adolescente grávida, que eu só fiquei sabendo depois que ganbou (ACS 1 , grupo A, $1^{\circ}$ encontro).

(...) como trabalhar esse menino que não tem uma família, né? Que não tem um apoio e que vê o tráfico como muitas vezes o apoio (...) eu cheguei pra um menininho que tem 11 anos, "Vamos brincar?", ele me olhou tipo "Tia, acorda, eu nunca brinquei na minha vida", ai eu "Então, vamos conversar". Então, fui lá entender o que era as drogas que ele mexia, pra poder ter a linguagem dele (...) (Psicóloga do CAPS, grupo B, $1^{\circ}$ encontro).

A dificuldade de comunicação e articulação com setores fora da área da saúde foi considerada importante, sendo exemplificada pela relação com o Centro de Referência em Assistência Social (CRAS):

(...) a gente não tem conhecimento do que exatamente o (...) CRAS faz, quando eles têm alguma demanda, alguma coisa que eles julgam que é da saúde, aí eles passam pra nós, mas, assim, a gente não tem essa relação do que fazem, do que não fazem (ACS 3, $4^{\circ}$ encontro, grupo A).

Relataram-se experiências de inserção de atividades dos membros da ESF na escola:

(...) eu faço estágio de promoção em saúde na comunidade, então é um estágio que é optativo na faculdade e ai eu escolbi fazer ele (...) a gente trabalbou habilidades de vida com as crianças aqui da (nome da escola). (...) a gente usou muito questão lúdica com eles, né, e trabalhar questão de respeito, de reconbecimento de espaço, do próprio autoconhecimento, a gente fez uma atividade com muita interação (...) (Técnica em enfermagem, $2^{\circ}$ encontro, grupo B).

Esse aspecto ilustra o reconhecimento da importância de ações intersetoriais para o trabalho das equipes da APS em relação à SMCA.

\section{O APOIO MATRICIAL COMO DISPOSITIVO TECNOLÓGICO: DESVELANDO PRÁTICAS INSTITUIÍDAS}

O dispositivo do AM possibilitou a identificação e problematização de diferentes perspectivas do cuidado realizado em SMCA:

(...) sobre a saúde mental, $80 \%$ dos cadastrados hoje fazem uso do CAPS, e eu creio que essa porcentagem seja realmente as pessoas que tomam remédios controlados ou pra dormir, ou pra tirar a ansiedade, crianças, muitas crianças, têm mais ou menos umas 7 crianças que frequentam a APAE [Associação de Pais e Amigos dos Excepcionais] (ACS 1, grupo B, $1^{\circ}$ encontro).

(...) o agente comunitário está fazendo papel de psicólogo nas casas. Você chega o pai tá com problema, a mãe tá com problema, ele conta tudo pro agente comunitário, problema de casa, brigas de casal, essas coisas, tudo (ACS 2, grupo B, $1^{\circ}$ encontro).

A própria discussão sobre o significado do AM culminou na reflexão sobre o cuidado em SMCA, a corresponsabilização dos serviços e os atravessamentos histórico-culturais.
(...) eu acho que teria que começar daqui pelo fato de que, querendo ou não quem pega primeiro somos nós (...). É que, então, a atenção básica sendo Saúde da Família não é só encaminhar (...). Então, às vezes, nós estamos pecando um pouco nisso também (...) tá sendo acompanhado lá, mas tá sendo acompanhado aqui também (...) (Enfermeira, grupo A, $2^{\circ}$ encontro, restituição).

(...) a gente não tem uma psicóloga aqui, eu não posso chamar as pessoas aqui e fazer uma reunião (...) como é que a atenção básica vai fazer? (...) mas tem que começar de algum lugar, eu acho que atenção básica não é lugar pra começar isso, tem que começar lá (faz referência ao CAPS) (...) eu não sou psicóloga, eu não entendo direito (...) (Técnica em enfermagem 1, grupo A, $2^{\circ}$ encontro, restituição).

(...) eu acho que essa dificuldade com a saúde mental é meio custoso, né, vem de todo um preconceito que se tinha na saúde mental antigamente, de isolamento (...). Às vezes, a gente não para pra escutar, na correria, o tanto de serviço que a gente tem, às vezes você tá aferindo uma pressão, é o momento que você poderia escutar o paciente, tem uma vacina esperando, tem um curativo esperando, né(...) e eu acho que, nesse corre corre, a gente peca um pouco sim (...) (Enfermeira, grupo A, $2^{\circ}$ encontro, restituição).

$\mathrm{O} \mathrm{AM}$ em ato provocou, no decorrer das reflexões, a ruptura do modo instituído da prática e a ressignificação de conceitos, como demonstrado nas seguintes falas:

(...) os termos existem, mas é uma realidade que não condiz com o que a gente vive (...) a gente não faz apoio matricial, a gente não faz educação permanente e se você pegar você vai ter lista, palestra montada (...) a gente é muito presa ainda no modelo biomédico, é muito fácil eu dar um CID [Código Internacional de Doença], tratar esse CID do que realmente promover algo para essa pessoa (Técnica de enfermagem, grupo B, $4^{\circ}$ encontro).

(...) é importante porque agora eu consigo ligar aqui no PSF [Programa Saúde da Família] e falar para as meninas "Olha, você me ajuda com fulaninho?" (...) hoje eu estava fazendo o acolbimento, ai eu falei assim "Qual é o seu PSF? Então, é interessante nesse sentido por você saber onde você pode ter o recurso” (...) (Psicóloga, grupo B, $6^{\circ}$ encontro, encontro de restituição).

(...) quando eu comecei a pensar nesse projeto e a questão era essa mesmo, que a gente está com muita demanda (referência aos serviços especializados em saúde mental) $e$ a atenção básica tem que tomar a responsabilidade, mas era mais uma questão de compartilhar (...) não passamos. A gente compartilha, faz junto, a gente precisa ser parceiro (...) mas isso foi um processo difícil de analisar, porque, até chegar nessa coisa de que "Olha, na verdade, eu queria passar o problema", não foi fácil, é uma coisa que vem com tempo e a gente precisa se permitir analisar, por isso épreciso questionar (...) (Pesquisadora, grupo A, $6^{\circ}$ encontro, restituição).

Como ilustram as falas acima, além do desconhecimento sobre o que os serviços do território fazem, há a necessidade de se discutir a diferença entre passar os casos e de compartilhar cuidados.

\section{DISCUSSÃO}

Os princípios norteadores da Socioclínica Institucional foram considerados de maneira interligada, permeando todo 
o processo da intervenção, balizando o dispositivo do AM. Para fins didáticos, a discussão dos resultados irá sinalizar cada princípio e sua contribuição para a prática do AM, contrastando também com a literatura científica atual.

O AM foi considerado um dispositivo analisador, fundamentando-se no princípio trabalho dos analisadores. $\mathrm{O}$ analisador deflagra e sustenta a análise na e da intervenção, possibilitando a explicitação das contradições, conflitos, desejos ${ }^{(9)}$, e sua função é desestabilizar o instituído. Dessa forma, permite que os agentes sociais olhem para sua prática ${ }^{(8)}$.

Um dos objetivos da Análise Institucional é entender uma determinada realidade social e organizacional por meio dos discursos e práticas de seus sujeitos ${ }^{(15)}$. Os sujeitos são, para a Análise Institucional, os agentes, todas as pessoas que falando ou se calando movimentam e mobilizam as instituições. Por isso, as instituições falam através de nós ${ }^{(9)}$.

Assim, tomou-se o dispositivo de AM como um analisador, ao propiciar o encontro entre os trabalhadores da ESF do CAPS e a pesquisadora, além de favorecer relatos de vivências e análises coletivas sobre a SMCA, pelos quais se explicitaram as instituições que os atravessam.

Para a Análise Institucional, as “... instituições se interpenetram e se articulam entre si para regular a produção e a reprodução da vida humana sobre a terra e a relação entre os homens" ${ }^{(9)}$. Como exemplo, cita-se a saúde como uma instituição que apresenta normas/regras visíveis (portarias, resoluções) e invisíveis (as concepções que estão presentes no processo saúde-doença, o modo como os profissionais colocam em prática o que está definido nas portarias). A Instituição Saúde também atravessa as relações entre os agentes sociais, bem como normatiza a maneira que as pessoas consideradas saudáveis convivem com as que são consideradas doentes; regula as relações profissionais, sendo atravessada por outras instituições, como a divisão técnica e social do trabalho, a educação, a pesquisa, a política-partidária ${ }^{(9,16)}$.

No desenvolvimento deste dispositivo de AM, procurou-se viabilizar que os participantes falassem sobre sua prática cotidiana. $O$ propósito foi permitir a participação dos sujeitos no dispositivo(8). Esse processo pode ocorrer de maneiras diferentes, mas, neste estudo, deu-se sob a forma de "sessões de grupo com objetivo analítico"(8), proporcionando um espaço de troca de experiências, discussão de casos e situações de saúde da população atendida na ESF, propiciando aos trabalhadores o questionamento de suas práticas e reflexões sobre novos modos de agir.

Discutir e problematizar os modos como os profissionais estão cuidando viabiliza a aprendizagem sobre e a partir do trabalho, fator que vem ao encontro da perspectiva da educação permanente em saúde ${ }^{(17)}$. Dessa maneira, os participantes e a pesquisadora foram colaboradores diretos no processo de construção coletiva do conhecimento sobre a produção do cuidado em SMCA, colocando em ato a intenção da produção do conbecimento.

Espaços para discussões e análises coletivas com as equipes de saúde podem ser utilizados como estratégia para fortalecimento da integração de seus membros, otimizando a comunicação e elaboração de intervenções ${ }^{(18)}$. Esses espaços minimizam as consequências da instituição divisão técnica e social do trabalho ${ }^{(9)}$, como a fragmentação do processo de cuidar.

Os relatos mostram as dificuldades de os profissionais da ESF assumirem a escuta, o que explicita o atravessamento da divisão técnica e social do trabalho. Esses ainda a consideram de maneira secundária em relação a outras formas de cuidar, como a realização de procedimentos. No entanto, a escuta é uma potente ferramenta de trabalho, que permite a criação do vínculo e olhar sensível ${ }^{(19-20)}$.

Nota-se, também, no estudo, um cuidado ainda pautado nas tecnologias duras ou uma tecnificação de tecnologias leves, fato apontado em outra pesquisa ${ }^{(21)}$, ao mostrar a prática do acolhimento de maneira tecnificada, com respostas prontas e com o processo de escuta voltado apenas às queixas. A prática de acolhimento realizada se justifica pela história, na institucionalização de saberes biológicos e de cuidados centrados no médico, especialmente curativos, representados como aqueles resolutivos.

O uso das tecnologias relacionais/leves ${ }^{(20)}$ permite aprofundar o entendimento da construção da subjetividade dos sujeitos, tecnologias importantes para o cuidado à população infantojuvenil. Como retratado nos resultados, inicialmente, os trabalhadores relataram certa aproximação com as crianças e adolescentes em seus atendimentos. Porém, a relação era frágil, muito submetida à mediação dos pais ou responsáveis.

Nesse sentido, é preciso olhar para a qualidade do vínculo que os profissionais de saúde apresentam junto ao público infantojuvenil. O ACS, por exemplo, apresenta um papel importante no acesso às famílias, às crianças e adolescentes, em decorrência de seu trabalho de inserção nos territórios de vida da população. Entretanto, mudanças nas atribuições desses profissionais na atual Política Nacional de Atenção básica ${ }^{(22)}$, assim como o incentivo a outras formas de organização da atenção básica que não contemplam os mesmos nas equipes, podem desencadear vínculos frágeis entre a população e o ACS, assim como com as equipes de saúde ${ }^{(23)}$.

Nos casos de intenso sofrimento psíquico, como de crianças e adolescentes com problemas de uso e abuso de drogas, o vínculo é um fator substancial. Abordar a situação do uso de drogas é algo sempre desafiador para os profissionais de saúde, como destacado nesta pesquisa pela fala da psicóloga do CAPS. Um estudo ${ }^{(24)}$ mostrou que as relações entre os profissionais e os adolescentes podem atuar como fator para melhor ou pior envolvimento no tratamento destes.

A temática das relações está no âmago do movimento da Reforma Psiquiátrica, com a adoção do modelo psicossocial exercido pelo CAPS e no modelo de atenção, adotado pela $\mathrm{ESF}^{(25)}$. Busca-se, em ambos os modelos, a problematização do conceito de processo saúde-doença e sobre o lugar dos sujeitos no processo de cuidar ${ }^{(25)}$.

Essa problematização permite a explicitação dos poderes, pois o usuário reconhece o poder do terapeuta, pois ele pensa precisar do saber especializado do mesmo, contudo reconhece o poder que também apresenta em seu processo de cuidado $^{(25)}$. A postura do profissional na lógica da atenção psicossocial e da ESF passa a ser de acompanhamento e não de prescrição de condutas ${ }^{(25)}$. Essa contratualidade é essencial no atendimento ao público infantojuvenil ${ }^{(4-5)}$. 
O cuidado em SMCA convoca um modo intersetorial de atuação; portanto, analisar como os setores se comunicam e articulam é necessário. Neste estudo, identifica-se pouca articulação entre os setores, sendo que os profissionais nem mesmo sabem a função de alguns equipamentos sociais do município, resultado também encontrado em outro estudo ${ }^{(5)}$. No entanto, há movimentos instituintes ${ }^{(9)}$, tal como a iniciativa de inserção na escola com o objetivo de vinculação com crianças e adolescentes, abordagem do autoconhecimento e habilidades de vida.

No decorrer da pesquisa, realizaram-se momentos de restituição pautados no princípio aplicação de modalidades de restituição. O momento da restituição seria a ocasião em que "supõe que se deva e se possa falar de algumas coisas que, em geral são deixadas à sombra. Essas coisas seriam comumente silenciadas, faladas apenas em corredores, cafés (...)"(12). Esses momentos permitiram a análise das transformações que ocorreram no desenvolver da pesquisa, com a problematização coletiva sobre a prática do AM. As restituições permitiram reflexões sobre o cuidado em saúde e a atenção aos contextos e interferências institucionais como a Instituição Psiquiátrica e a Instituição divisão técnica e social do trabalho, que atravessam o modo de cuidar dos trabalhadores.

Ao analisar a Instituição Saúde, nota-se, neste estudo, a expressão de um modo de cuidado pautado na psiquiatria biológica, no modelo centrado na figura do médico ou no especialista, com ações de saúde voltadas ao tratamento de doenças, ainda que a ESF e o CAPS tenham sido propostas de organização de serviços com o objetivo de reorientação do modelo de atenção à saúde. Esse fato sinaliza a possibilidade de que "as instituições frequentemente são capazes de produzir o oposto do propósito para o qual foram fundadas"(26).

As modificações que ocorreram no percurso da pesquisa foram analisadas nos momentos de restituição, estando interligados à análise da encomenda e das demandas ${ }^{(8)}$. Os participantes puderam olhar para sua prática e ressignificá-la, alterando, por exemplo, o modo de pensar a escuta. Aponta-se como ferramenta importante os momentos de restituição, pois proporcionaram ao grupo a análise sobre as transformações de suas percepções e práticas à medida da ocorrência dos encontros, e não apenas no final da pesquisa, promovendo uma participação mais singular dos sujeitos ${ }^{(27)}$.

Para o apoiador, a participação da equipe será sempre um desafio, pois é requerido que oportunize aos profissionais apresentarem seu ponto de vista sobre determinada situação. Durante esse processo, é necessário que o apoiador aceite analisar a opinião dos trabalhadores/equipe, mesmo que sejam diferentes e tenham uma direção contrária à sua compreensão sobre determinado tema.

Nesse sentido, a análise da implicação se mostra potente na prática do AM. O conceito de implicação para a Análise Institucional trata das relações que o pesquisador e os participantes de um estudo mantêm com a pesquisa em si (implicações primárias) e o envolvimento do pesquisador e participantes com outras instituições, como a instituição família, igreja, profissão, as crenças do pesquisador, relações ideológicas e libidinais, entre outras ${ }^{(8,28)}$.
No caso do AM, a análise da implicação auxilia o apoiador na análise de suas implicações, ou seja, do envolvimento, das relações que todos apresentam com o AM em si, como sobre os métodos utilizados no matriciamento (reunião, atendimento conjunto, discussão de caso etc.), o que se espera do matriciamento (compartilhamento ou transferência dos atendimentos) e as implicações secundárias relacionadas às temáticas ou linhas de cuidado abordadas.

Pode-se afirmar que foi realizada uma análise das implicações em algumas falas, como a da enfermeira do grupo $\mathrm{B}$, ao problematizar sua prática em relação à aprendizados anteriores no campo do cuidado à saúde mental e à própria forma como a sociedade dita esse cuidado. Considera-se que os cuidados em saúde mental sofrem interferências institucionais e estão centrados em duas instituições: a Psiquiatria e a Psicologia, sem considerar, muitas vezes, outras disciplinas e os cuidados integrais.

A mudança de pensamento da própria pesquisadora também explicita a potência da análise das implicações ${ }^{(28)}$. A mesma pôde olhar para seu lugar de enfermeira de um CAPS e de apoiadora/pesquisadora, entendendo que seu pensamento também estava atravessado pela segmentação do cuidado, entre o que é pertencente exclusivamente ao CAPS ou à ESF.

O dispositivo do AM, sustentado pela Socioclínica, possibilitou a análise e ressignificação do pensamento e prática profissional, da necessidade de compartilhamento do cuidado, do apoio no desenvolvimento de formas de cuidar que integrem ações de promoção, prevenção e tratamento no âmbito da $\mathrm{SMCA}^{(5-6)}$, além de trazer de volta à luz os motivos para os quais foi fundada (no sentido do mito fundador ou da profecia inicial) cada modalidade de serviço de saúde ${ }^{(29)}$.

\section{CONCLUSÃO}

Neste estudo, o AM favoreceu a desterritorialização dos profissionais, revelando como se dá o cuidado em SMCA praticado e os atravessamentos que ocorrem na produção deste, assim como possíveis caminhos a serem trilhados para aprimorar as ações de saúde.

Verificou-se que os trabalhadores da APS ainda se pautam no paradigma da psiquiatria biológica, limitando o cuidado e identificando-o como restrito à atuação de especialistas. Revelam-se pistas importantes para a área da enfermagem na experimentação do AM, assim como a possibilidade de um (re)olhar para a inserção e a prática da enfermagem no desenvolvimento do cuidado em SMCA. Ressaltam-se, principalmente, vivências importantes do AM em pontos de atenção à saúde, como a ESF e o CAPS, que apresentam o mandato social de reorganização dos modos de se cuidar e considerar o processo saúde-doença em todas suas dimensões.

Aponta-se como limitação deste estudo a não participação dos médicos nas discussões, o que revela o modo de funcionamento das equipes de saúde. Os enfermeiros, técnicos de enfermagem, ACS e odontólogos participam com mais frequência de ações de AM, educação permanente em saúde e reuniões de equipe. A não participação dos médicos 
pode dificultar processos de repensar as práticas e o trabalho interprofissional e colaborativo.

Os princípios da Socioclínica Institucional favoreceram o desenvolvimento do AM em SMCA na ESF, podendo funcionar como pistas para o exercício do AM em outros contextos e temáticas, contribuindo para a reorientação do processo de trabalho em saúde e a articulação entre equipes e serviços de saúde.

\section{RESUMO}

Objetivo: Analisar o apoio matricial para equipes da Estratégia Saúde da Família em relação à Saúde Mental em Crianças e Adolescentes Método: Pesquisa-intervenção de abordagem qualitativa, fundamentada pelo referencial da Análise Institucional, a socioclínica, realizada com dezoito trabalhadores de saúde de duas equipes da Estratégia Saúde da Família e do Centro de Atenção Psicossocial de um município de pequeno porte, localizado no interior do estado de São Paulo, por meio de onze encontros de reflexão. Resultados: Foram apresentados a partir dos temas: A dinâmica das relaçóes no território da ESF; O apoio matricial como dispositivo tecnológico: desvelando práticas instituídas. Posteriormente, foi realizada a discussão dos resultados a partir dos princípios da Socioclínica Institucional. Conclusão: O apoio matricial em saúde mental infantojuvenil, pautado no referencial da Socioclínica Institucional, favoreceu a desterritorialização dos profissionais, revelando como se dá o cuidado em saúde mental para crianças e adolescentes e os atravessamentos que ocorrem na produção desse cuidado, assim como possíveis caminhos a serem trilhados para aprimorar as ações de saúde.

\section{DESCRITORES}

Enfermagem; Saúde Mental; Estratégia Saúde da Família; Equipe de Assistência ao Paciente; Prática Profissional.

\section{RESUMEN}

Objetivo: Analizar la matriz de apoyo a los equipos de la Estrategia Salud de la Familia con relación a la Salud Mental en la Niñez y la Adolescencia. Método: Investigación-intervención con enfoque cualitativo, con base en el marco de Análisis Institucional, el socioclínico, realizado con dieciocho trabajadores de salud de dos equipos de la Estrategia Salud de la Familia y del Centro de Atención Psicosocial de una pequeña ciudad, ubicada en el interior del estado de São Paulo, a través de once encuentros de reflexión. Resultados: Se presentaron a partir de los temas: La dinámica de las relaciones en el territorio de la ESF; El soporte matricial como dispositivo tecnológico: revelando prácticas establecidas. Posteriormente, se discutieron los resultados con base en los principios de Socioclínicas Institucionales. Conclusión: La matriz de apoyo en salud mental infantil, con base en el marco Socioclínico Institucional, favoreció la desterritorialización de los profesionales, revelando cómo se brinda la atención en salud mental a la niñez y adolescencia y los cruces que se dan en la producción de este cuidado, así como en lo posible caminos a seguir para mejorar las acciones de salud.

\section{DESCRIPTORES}

Enfermería; Salud Mental; Estrategia de Salud Familiar; Grupo de Atención al Paciente; Práctica Profesional.

\section{REFERÊNCIAS}

1. Braga CP, Oliveira AF. Child and adolescent mental health policy: history and paths to participation. Ciênc Saúde Coletiva. 2019;24(2):401-10.

2. Brasil. Ministério da Saúde. Secretaria de Atenção à Saúde. Departamento de Atenção Especializada e Temática. Fórum Nacional de Saúde Mental Infantojuvenil: recomendações: de 2005 a 2012 [Internet]. Brasília, DF; 2014 [cited 2020 Feb 10]. Available from: http://bvsms. saude.gov.br/bvs/publicacoes/forum_nacional_saude_mental_infantojuvenil.pdf

3. Pigozi PL, Machado AL. Mapping the provision of care to an adolescent victim of school bullying by the Family Health Strategy in Brazil. Cienc Saúde Coletiva. 2020;25(1):353-63. https://doi.org/10.1590/1413-81232020251.04212018

4. Batista KA, Oliveira PR. The child mental health in primary care: reflections on care practices in the municipality of Horizonte-CE. Pesq Prat Psicossociais [Internet]. 2017 [cited 2020 Sept 18];12(3):1-17. [Portuguese]. Available from: http://pepsic.bvsalud.org/pdf/ppp/v12n3/06.pdf

5. Teixeira MR, Couto MC, Delgado PG. Primary care and collaborative care in children and adolescents psychosocial interventions: facilitators and barriers. Cienc Saúde Coletiva. 2017;22(6):1933-42. https://doi.org/10.1590/1413-81232017226.06892016

6. Campos GW, Domitti AC. [Matrix support and reference team: a methodology for interdisciplinary health work management]. Cad Saude Publica. 2007;23(2):399-407. Portuguese. https://doi.org/10.1590/S0102-311X2007000200016

7. Penido CM, Alves M, Rosangela SR, Fátima FM. Matrix support as technology in health. Saúde Debate [Internet]. 2010 [cited 2020 Feb 15];34(86):467-74. [Portuguese]. Available from: https://www.redalyc.org/pdf/4063/406341769009.pdf

8. Monceau G. A Socioclínica institucional para pesquisas em educação e em saúde. L'Abbate S, Mourão LC, Pezzato LM, editores. Análise institucional \& saúde coletiva. São Paulo: Hucitec; 2013. p. 91-103.

9. Baremblitt GF. Compêndio de análise institucional e outras correntes: teoria e prática. 6th ed. Belo Horizonte: Instituto Felix Guattari; 2012.

10. Fortuna CM, Silva SS, Mesquita LP, Matumoto S, Oliveira PS, Santana FR. The institutional socio-clinic as a theoretical and methodological framework for nursing and health research. Texto Contexto Enferm. 2017;26(4):1-8. https://doi.org/10.1590/0104-07072017002950017

11. Monceau G, Soulière M. Mener la recherche avec les sujets concernés: comment et pour quels résultats? Educ Socialisation. 2017; 45:1-16. https://doi.org/10.4000/edso.2525

12. Lourau R. René Lourau na UERJ: análise institucional e práticas de pesquisa. Rio de Janeiro: NAPE/UERJ; 1993.

13. Bourrassa B, Picard F, Le Bossé Y, Fournier G. Accompagner les groupes de recherche collaborative: en quoi consiste ce "faire avec"? Phronesis. 2017;6(1-2):60-73. https://doi.org/10.7202/1040218ar

14. Paillé P, Mucchielli A. L'analyse qualitative en sciences humaines et sociales. 3rd ed. Paris: Armand Colin; 2012. https://doi.org/10.3917/ arco.paill.2012.01

15. Spagnol CA, L'Abbate S, Monceau G, Jovic L. [Socianalytical device: intervention instrument and data collection in qualitative research in nursing]. Rech Soins Infirm. 2016;124(124):108-17. French. https://doi.org/10.3917/rsi.124.0108 
16. Silva MA, Abrahão AL. [Comprehensive Care Policy for users of alcohol and other drugs: a narrative-guided analysis]. Interface (Botucatu). 2020;24:e190080. Portuguese. https://doi.org/10.1590/interface.190080

17. Rojas FL, Kehrig RT, Biato EC, Santos NC. [Permanent education in health: the rethinking about the construction of health practices]. J Health NPEPS. 2019;4(2):310-30. Portuguese. https://doi.org/10.30681/252610103730

18. Peruzzo HE, Bega AG, Lopes AP, Haddad MC, Peres AM, Marcon SS. The challenges of teamwork in the family health strategy. Esc Anna Nery. 2018;22(4):e20170372. https://doi.org/10.1590/2177-9465-ean-2017-0372.

19. Santos AB. Escuta qualificada como ferramenta de humanização do cuidado em saúde mental na Atenção Básica. Rev Rede APS. 2019;1(2):170-9. https://doi.org/10.14295/aps.v1i2.23

20. Campos DB, Bezerra IC, Jorge MS. Mental health care technologies: primary Care practices and processes. Rev Bras Enferm. 2018;71(5 suppl 5):2101-8. https://doi.org/10.1590/0034-7167-2017-0478

21. Fortuna CM, Oliveira KF, Feliciano AB, Silva MV, Borges FA, Camacho GA, et al. Embracement as an analyzer of the relationships between professionals, managers and users. Rev Esc Enferm USP. 2017;51:e03258. https://doi.org/10.1590/s1980-220x2016003303258

22. Giovanella L, Franco CM, Almeida PF. National Primary Health Care Policy: where are we headed to? Cienc Saúde Coletiva. 2020;25(4):1475-81. https://doi.org/10.1590/1413-81232020254.01842020

23. Morosini MV, Fonseca AF. Community workers in Primary Health Care in Brazil: an inventory of achievements and challenges. Saúde Debate. 2018;42(n spe 1):261-74. https://doi.org/10.1590/0103-11042018s117

24. Gonçalves JR, Canassa LW, Cruz LC, Pereira AR, Santos DM, Gonçalves AR. Adherence to treatment: perception of adolescents in chemical dependency. Rev Latino-Am Enfermagem. 2019;15(1):57-63. https://doi.org/10.11606/issn.1806-6976.smad.2019.000415

25. Yasui S, Luzio C, Amarante P. [Psychosocial care and primary care: life as territory in the field]. Rev Polis Psique. 2018;8(1):173-90. Portuguese. https://doi.org/10.22456/2238-152X.80426

26. Fortuna CM, Monceau G, Valentim S, Mennani KL. [Socio-clinical research in France: notes on institutional analysis]. Fractal Rev Psicol. 2014;26(2):255-66. Portuguese. https://doi.org/10.1590/1984-0292/1309

27. Santana FR, Fortuna CM, Monceau G. Promotion de la santé et prévention des maladies dans la formation professionnelle en soins infirmiers au Brésil et en France. Revue Éducation Santé Societes [Internet]. 2017 [cited 2020 Apr 14];3(2):115-35. Available from: https://www.researchgate.net/publication/322488891/download

28. Londero M, Soares L. [Analysis of Implications: a problematizing power to the researchers supposedly "known"]. Rev Polis Psique. 2018;8(1):144-63. Portuguese. https://doi.org/10.22456/2238-152X.80424.

29. Sada D. Le moment agon. Cahiers I'implication [Internet]. 2001 [cited 2020 Apr 14];(4):29-33.Available from: http://cahiers.implication. free.fr/numero4.html 